\title{
RAPIDLY GRASPING LEARNERS' MATHEMATICAL PERFORMANCE FROM BRAIN ACTIVITY
}

\author{
Kodai Suzuki, Satoshi Yazawa, Reiko Matsunaka, Toru Iwasawa and Kazuo Hiraki \\ Graduate School of Art and Science, University of Tokyo \\ 3-8-1, Komaba, Meguro-ku, Tokyo, Japan
}

\begin{abstract}
For most people, it takes a lot of time to acquire mathematical skills. Mathematical problems are complex because they consist of many steps. Therefore, it is not easy to grasp the learners' performance. It is ideal to know the level of learners in a reasonably short time. In this experiment, we aimed to grasp the learners' performance from short time solving time estimation for the mathematical problems. Participants performed the task of estimating the solving time of mathematical problems. Time frequency analysis was conducted from Electroencephalogram (EEG) recordings. As a result, people with well mathematical performance had higher theta activity than those with poor performance. In conclusion, the present research suggests that brain activity measurement during solving time estimation can be effective in grasping the learners' level in a short time.
\end{abstract}

\section{KEYWORDS}

Mathematical Education, Passive BCI, Problem Solving, Educational Technology, EEG, Time Frequency Analysis

\section{INTRODUCTION}

Many people spend so long time to improve their mathematical skills. Because the cognitive processes involved in mathematical problem solving are very complex, it is very difficult to find an efficient learning method. It is important to be able to diagnose learners' performance rapidly for the efficient improvement of mathematical skills. Passive brain computer interface (passive BCI), which captures the mental state of healthy users (Zander \& Kothe, 2011), is a technology of interest and could be effective in achieving this end.

In this experiment, in order to measure brain activity, we used Electroencephalogram (EEG). EEG has superb temporal resolution (Martinez et al., 2004). Using EEG, we can examine brain activity by isolating the epochs during solving time estimation for the mathematical problem from other brain activities. Theta activity has been linked to long-term memory, leading to faster problem solving (Lin et al., 2012). Resolving the problem from the fact retrieval showed higher theta activity than based on insights (Grabner \& De Smedt, 2011). However, previous studies have dealt with simple problems such as arithmetic operations, so there was a challenge that multiple processes such as making plans and implementation could not be separated.

In the present research, our goal is to grasp the learners' performance in a short time when the learners are performing meta-cognition, focusing on understanding and planning. In the present research, participants performed a sort of meta-cognition task to estimate their solving time. We examined whether brain activity, especially theta power, was related to learning performance.

\section{METHOD}

\subsection{Participants}

Fourteen high school students participated in this experiment (range: 15-16, 12 males and 2 females). Every participant is paid 2000 yen equally. We have obtained the consent of the participants and their parents. We 
conducted this experiment with the consent of the Ethics Review Committee of university of Tokyo. Each participant submitted a written consent.

\subsection{Procedure}

\subsubsection{Two Phase: Estimating the Problem and Implementing the Problem}

The experiment consists of two parts. These two parts were performed on the same day. The experimental stimulus was created using E-Prime, a software for EEG experiments. In the first phase, we used 40 mathematical problems from a high school textbook. The mathematical problem is presented for ten seconds on the display in front of the participant. Participants are required to think about how to solve the presented problems. After the problem was presented, participants answer two questions. First, they give the solving time estimation. Second, they give the degree of certainty (confidence) that they can solve the problem. Participants answer each of the two questions within three seconds. The problems are presented in random order.

In the phase of implementing the problem, we used five mathematical problems. Participants entered their answers in the iPad answer recording app with an Apple pencil. Problems are presented one by one, and participants take a break after solving each problem. The time limit for solving the problem is five minutes. If the participant can solve the problem, the problem solving can be terminated by key input. After completing the problem solving, the participant answers the confidence within three seconds. Five problems are extracted from those presented in the previous phase. Each participant solves the same problems. Problems are presented in random order.

\subsubsection{EEG Recording and EEG Analysis}

EEG measurement provides a method to non-invasively determine the voltage at the electrode site and investigate the brain activity. We used 128-channel HCGSN v.1.0 (Geodesics, 2007). The sampling rate was $250 \mathrm{~Hz}$. The ground electrode was placed at the position of $\mathrm{Cz}$. Reference electrodes was placed at the right and left ears. The impedance was maintained below $100 \mathrm{k} \Omega$ throughout the recordings.

In the analysis, we excluded one participant from the EEG analysis due to a failure of the experiment. One epoch is eleven seconds, one second fixation and ten seconds problem presentation in the phase of estimating the problem. The raw EEG signal was filtered using a bandpass filter with a 1-40 $\mathrm{Hz}$ passband. We analyzed the right parietal region, using four electrodes. In time frequency analysis, we apply the fast Fourier transform with a 0.5 second Hanning window. The baseline is the one second fixation interval before the stimulus is presented.

Based on the performance in the phase of implementing the problems, participants were divided into two groups. One point was given for solving one problem, and participants who got 0-1points are classified as the poor group (seven people), and participants who got 2-4 points are classified as the well group (six people). Based on the results of the Smirnov-Grubbs test, one participant of the poor group was excluded.

\section{RESULT}

We obtained the time frequency pattern of participants during solving time estimation

Figure 1.A shows the average time frequency patterns of those with well performance and those with poor performance in the phase of estimating the problem. Theta band $(5 \mathrm{~Hz})$ of the participant with the well performance showed higher activity. In particular, a significant difference was observed during the time period of 3.6-3.8s after the stimulus presentation (paired t-test, $\mathrm{p}<0.05$ ). Moreover, figure 1.B shows that participants with well performance showed higher theta activity over the head. 
A

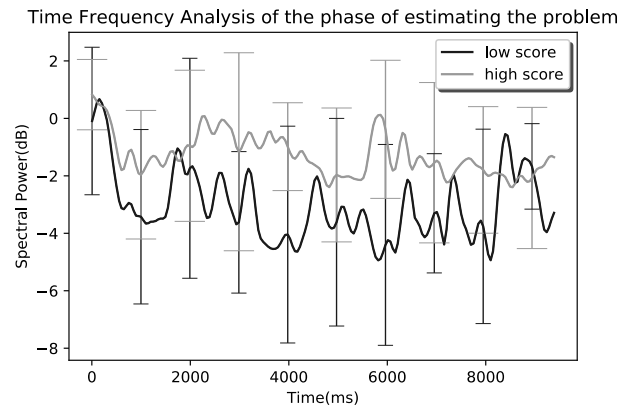

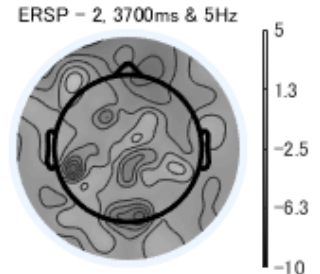

(a)

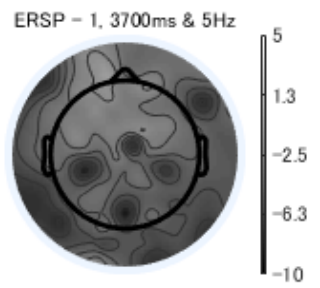

(b)

Figure 1. A: This shows the average time frequency patterns $(5 \mathrm{~Hz}$; theta wave) of those with well performance and those with poor performance in the phase of estimating the solving time. B: (a) is a topography of theta wave of a person with well performance, and (b) is a topography of theta wave of a person with poor performance

\section{DISCUSSION}

People with well mathematical performance had more theta activity than those with poor performance. Theta activity are associated with the retrieval of facts (Grabner \& De Smedt, 2011). People with well mathematical performance are expected to have more mathematical knowledge. Based on the visualization of the topography, theta activity occurs across multiple sites. This result was consistent with the finding that memory and learning act as a whole rather than working separately (Başar, 2005). The results obtained here provide important insights in constructing passive BCI for education.

\section{CONCLUSION}

The present research focused on the brain activity and meta-cognition on the complex mathematical problems. Participants with well mathematical performance showed higher theta activity. This result suggests that it is possible to predict the learners' performance in a short time from the brain activity during solving time estimation for the complex problems. Since the estimation for the solving time may differ depending on the type and content of the problem, it is important to conduct experiments that control the type of mathematical problems in the future.

\section{ACKNOWLEDGEMENT}

This work was supported by CREST, JST and SIP, Japan.

\section{REFERENCES}

Başar, E., 2005. Memory as the "whole brain work": a large-scale model based on "oscillations in supersynergy". International Journal of Psychophysiology, 58(2-3), pp.199-226.

Geodesics, E., 2007. Geodesic Sensor Net Technical Manual. Eugene: Electrical geodesics.

Grabner, R.H. and De Smedt, B., 2011. Neurophysiological evidence for the validity of verbal strategy reports in mental arithmetic. Biological psychology, 87(1), pp.128-136.

Lin, C.L. et al, 2012, August. Brain dynamics of mathematical problem solving. In 2012 Annual International Conference of the IEEE Engineering in Medicine and Biology Society (pp. 4768-4771). IEEE.

Martınez-Montes, E. et al, 2004. Concurrent EEG/fMRI analysis by multiway partial least squares. NeuroImage, 22(3), pp.1023-1034.

Zander, T. O. and Kothe, C., 2011. Towards passive brain-computer interfaces: applying brain-computer interface technology to human-machine systems in general. Journal of neural engineering, 8(2), 025005. 